\title{
Transbronchial needle aspiration in sarcoidosis: Yield and predictors of a positive aspirate
}

\author{
Rocco Trisolini, MD, ${ }^{a}$ Carmine Tinelli, MD, ${ }^{d}$ Alessandra Cancellieri, MD, ${ }^{b}$ Daniela Paioli, MD, ${ }^{a}$ Marco Alifano, MD, ${ }^{\mathrm{c}, e}$ \\ Maurizio Boaron, MD, ${ }^{\mathrm{c}}$ and Marco Patelli, MD ${ }^{\mathrm{a}}$
}

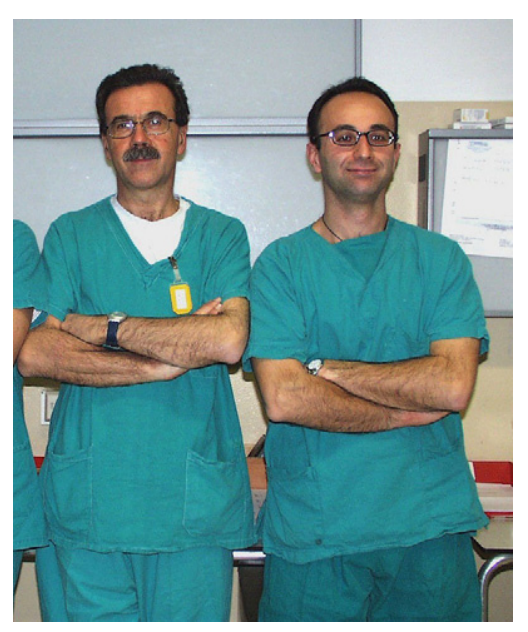

Drs Patelli (left) and Trisolini (right)
Objective: Transbronchial needle aspiration is a useful diagnostic procedure in sarcoidosis, but widely variable yields are reported. This study determined the diagnostic contribution of standard transbronchial needle aspiration in a large series of patients with sarcoidosis and evaluated predictor variables that might influence its results.

Methods: Sixty-one consecutive patients with suspected sarcoidosis in a 2-year period were prospectively enrolled and underwent standard transbronchial needle aspiration with a 19-gauge needle. The following predictor variables were recorded for each patient: age; sex; sarcoidosis stage; operator; size, location, and number of sampled lymph nodes; number of needle passes per sampled node; and adequacy of both histologic and cytologic transbronchial needle aspiration specimens.

Results: Sarcoidosis was diagnosed in 53 patients. Lymph node aspiration biopsy was successfully achieved in 50 of 53 cases (94\%). Nonnecrotizing epithelioid granulomas were observed in 42 of 53 patients (79\%), with similar results for stage I (27/ $33,82 \%)$ and stage II $(15 / 20,75 \%)$ disease. Sampling of two lymph node stations was the only variable significantly associated with a likelihood of a sarcoidosispositive aspirate or biopsy sample in both univariate (odds ratio $0.15,95 \%$ confidence interval $0.02-0.79$ ) and multivariate (odds ratio 0.12 , 95\% confidence interval $0.02-$ $0.70)$ analyses.

Conclusion: Standard transbronchial needle aspiration allows successful lymph node sampling in nearly all patients with sarcoidosis and is associated with high diagnostic yield regardless of disease stage. Whenever possible, sampling of more than one nodal station is advised to increase diagnostic yield. Mediastinoscopy should be reserved for patients with negative transbronchial needle aspiration findings.

From the Units of Thoracic Endoscopy and Pulmonology, ${ }^{\mathrm{a}}$ Pathology, and Thoracic Surgery, ${ }^{c}$ Maggiore Hospital, Bologna, Italy; Clinical Epidemiology and Biometry Service, IRCCS Policlinico San Matteo, Pavia, Italy; and the Thoracic Surgery Unit, Hôtel-Dieu Hospital, AP-AH, Paris, France. ${ }^{\mathrm{e}}$

Received for publication July 28, 2007; revisions received Oct 29, 2007; accepted for publication Nov 13, 2007.

Address for reprints: Rocco Trisolini, MD, Thoracic Endoscopy and Pulmonology Unit, Maggiore Hospital, Largo B. Nigrisoli 2, 40133 Bologna, Italy (E-mail: rocco. trisolini@ausl.bologna.it).

J Thorac Cardiovasc Surg 2008;135:837-42 0022-5223/\$34.00

Copyright (C) 2008 by The American Association for Thoracic Surgery

doi:10.1016/j.jtcvs.2007.11.011
$\mathrm{M}$ ediastinoscopy is associated with the best diagnostic yield in the pathologic confirmation of sarcoidosis with mediastinal involvement (stages I and II of the disease). ${ }^{1}$ Nonetheless, risk-benefit and cost-benefit analyses, ${ }^{2}$ as well as the development of transbronchial needle aspiration (TBNA), a minimally invasive bronchoscopic procedure that allows aspiration from the mediastinum, have markedly decreased the use of mediastinoscopy in this setting.

TBNA, developed for use with flexible bronchoscopy in the 1980s, was mainly used for the diagnosis and staging of lung cancer. ${ }^{3}$ In this specific setting, several studies evaluated a number of factors to explain the differences seen among the reported diagnostic yields of the procedure and found several predictors of a positive TBNA aspirate, such as high prevalence of lymph node metastasis in the study population, ${ }^{4,5}$ increasing lymph node size, ${ }^{6,7}$ right paratracheal and subcarinal locations, ${ }^{6,8}$ use of a histology needle, ${ }^{9}$ use of rapid on-site cytopathologic examination (ROSE), ${ }^{7,10}$ and small cell carcinoma histologic type. ${ }^{6,7}$

No such studies are available for use of TBNA in the setting of sarcoidosis. Furthermore, the few studies that have specifically addressed the diagnostic value of TBNA in sarcoidosis, predominantly retrospective, have reported highly variable 


\author{
Abbreviations and Acronyms \\ CT = computed tomography \\ ROSE $=$ rapid on-site cytopathologic examination \\ TBLB $=$ transbronchial lung biopsy \\ TBNA $=$ transbronchial needle aspiration
}

yields. ${ }^{11-16}$ The likelihood of a diagnostic TBNA was much higher in patients with stage I than stage II disease in most studies, ${ }^{12-15}$ and some authors have hypothesized that stage I lymph nodes could have a higher density of granulomas. ${ }^{11}$ We performed a prospective study to determine more precisely the diagnostic contribution of standard TBNA in cases of sarcoidosis and to evaluate factors possibly influencing its results.

\section{Materials and Methods \\ Study Design}

This study was approved by the ethics committee of the Azienda USL di Bologna, and all study participants provided written, informed consent. Consecutive patients referred to the Thoracic Endoscopy Unit of Maggiore Hospital (Bologna, Italy) for pathologic confirmation of suspected sarcoidosis with hilar or mediastinal involvement (stage I and II) between January 1, 2004, and December 31,2005 , were prospectively studied.

Because presentations of sarcoidosis vary enormously, the expectation of the disease was based on a variable combination of clinical and imaging findings. Clinically, nonspecific constitutional manifestations (fever, fatigue, malaise, weight loss), respiratory symptoms (especially cough and dyspnea), erythema nodosum, arthralgias, palpable peripheral enlarged lymph nodes, and findings related to specific organ involvement (eg, uveitis, skin lesions) were carefully evaluated. Imaging studies included both chest radiography and contrast-enhanced computed tomography (CT) in each case, but radiographic staging of the disease was based on the posteroanterior chest radiograph, as universally accepted. ${ }^{17}$

After CT scan, each patient underwent bronchoscopy with TBNA, transbronchial lung biopsy (TBLB), and bronchoalveolar lavage. The following variables were recorded for each patient: age; sex; sarcoidosis stage; operator performing the procedure; size, location, and number of sampled lymph nodes; number of needle passes for each sampled lymph node; and adequacy of cytologic and histologic TBNA specimens.

Patients with inconclusive results of bronchoscopic procedures underwent mediastinoscopy. For those who refused surgery, accurate clinical and radiologic follow-up were carried out. Patients ultimately found not to have sarcoidosis were excluded from the analysis.

Two well-trained bronchoscopists (MP and RT) performed TBNAs during flexible bronchoscopy with a 19-gauge histology needle (MW-319; ConMed Endoscopic Technologies, Billerica, Mass) according to a previously described technique., ${ }^{8,18}$ Before any other sampling procedures, one to three needle passes were performed on one or two stations of the American Thoracic Society classification scheme ${ }^{19}$; in most patients who had a single node station punctured, three needle passes were performed. Only lymph nodes with a short axis greater than $1 \mathrm{~cm}$ at CT scan were selected for aspiration. The location and the number of stations to be sampled in the individual patient were left to the discretion of each operator, but preference was given to enlarged nodes in the right paratracheal and subcarinal stations, because TBNA in those areas was consistently associated with the highest yield in the settings of both neoplastic and granulomatous disorders. ${ }^{6,8,15,16,20,21}$ All TBNA specimens from a single site were pooled as a combined specimen.

\section{Handling of Aspirates and Pathologic Categorization of TBNA Samples}

TBNA specimens were collected on clean glass slides. The histologic core of the tissue was gently removed from the slide and placed in a formalin solution. The cytologic material was smeared and subsequently stained with May-Grünwald Giemsa, Papanicolaou, and Ziehl-Neelsen stains.

Pathologists blinded to the patient's clinical and radiologic details performed the categorization of TBNA samples. The presence of material consistent with the architecture of lymph nodes (histologic samples) or the presence of a preponderance of lymphocytes (cytologic samples) defined the adequacy of TBNA. A diagnostic sample, histologic or cytologic, contained nonnecrotizing epithelioid granulomas in the absence of identifiable foreign-body reactions or positive stains for acid-fast bacilli and fungi. ${ }^{17,22,23}$

\section{Statistical Analysis}

Patients were compared for aspirate status (positive or negative) with the use of the Mann-Whitney test for continuous variables and the $\chi^{2}$ test or the Fisher test as appropriate for categoric or nominal variables. Associations between aspirates status and predictor variables are reported as odds ratio (OR) with $95 \%$ confidence interval (CI) and were evaluated with logistic regression model. Only significant variables in univariate analysis (.2 level) or those deemed clinically important were included in the multivariate model. Analyses were performed with Statistica for Windows Software (StatSoft Inc, Tulsa, Okla) and with the STATA software package (Stata Corporation, College Station, Tex).

\section{Results}

During the study period, sarcoidosis was diagnosed in 53 of 61 patients who met the inclusion criteria. In the remaining 8 cases, other causes were responsible for the clinical and radiologic findings (4 cases of lymphoproliferative disorders, 3 cases of mycobacterial infection, and 1 case of pneumoconiosis).

The final diagnosis was established on the basis of the results of the bronchoscopic sampling procedures in 50 of 61 cases (sarcoidosis in 47 patients and mycobacterial infection in 3 patients), on the results of mediastinoscopy in 6 cases (sarcoidosis in 2 patients and lymphoproliferative disorders in 4 patients), and on the evaluation of current and followup clinicoradiologic data in 5 cases (sarcoidosis in 4 patients and pneumoconiosis in 1 patient).

Most patients with sarcoidosis in this series were initially seen with a variable combination of nonspecific constitutional manifestations, respiratory symptoms, erythema nodosum, and arthralgias. In a few cases, the possibility of sarcoidosis 
was raised with respect to a symptom-free patient on the basis of results of imaging studies performed for unrelated reasons (chest radiography in the diagnostic workup preceding surgery).

The 53 sarcoidosis patients were all white, had a mean \pm SD age of $44.7 \pm 12.7$ years, and had a male/female ratio of 0.7. In 47 of $53(89 \%)$, the definitive diagnosis was obtained with the help of bronchoscopy procedures, whereas in 6 cases it was achieved by either mediastinoscopy $(n=2)$ or clinicoradiologic follow-up $(n=4)$. With respect to the bronchoscopic procedures, the results of TBNA (overall 79\%, stage I $82 \%$, stage II $75 \%$ ) exceeded those of TBLB (overall $55 \%$, stage I $41 \%$, stage II $79 \%$ ), although the best diagnostic yield was obtained by combining the two methods (overall $89 \%$, stage I $88 \%$, stage II $90 \%$ ). In 18 of 53 cases, the diagnosis of sarcoidosis was established exclusively by TBNA (34\% increase in diagnostic rate), whereas TBLB was the only means of diagnosis in 5 of 53 cases (9\% increase in diagnostic rate).

Table 1 shows the patient-based analysis of TBNA results in the 53 patients with sarcoidosis. TBNA allowed material representative of lymph node tissue to be obtained in 50 of 53 cases (94\%). Nonnecrotizing epithelioid cell granulomas were observed in TBNA samples in 42 of 53 cases (79\%), without differences between stages I $(27 / 33,82 \%)$ and II $(15 / 20,75 \%)$.

The mean \pm SD size of sampled lymph nodes was $1.85 \pm$ $0.43 \mathrm{~cm}$. The diagnostic yield was significantly higher in patients who had two lymph nodes sampled relative to those with only one sampled $(25 / 27,92.5 \%$, vs $17 / 26,65 \%$, respectively, $P=.01$ ).

Table 2 illustrates the results of the 80 TBNA procedures performed on all the patients included according to the lymph node station sampled. Sampled lymph nodes were located in the following stations of the American Thoracic Society classification scheme ${ }^{19}$ : subcarinal $(\mathrm{n}=41)$, right paratracheal $(\mathrm{n}=30)$, right hilar $(\mathrm{n}=8)$, and left paratracheal $(\mathrm{n}=1)$. Histologic tissue was obtained from 48 of 80 sampled lymph nodes, whereas cytologic material was retrieved from 80 of 80 nodes. The percentages of both adequate and diagnostic cytologic TBNA samples exceeded the percentages of histologic ones $(87.5 \%$ and $70 \%$, respectively, vs $36 \%$ and $22.5 \%$, respectively).

Minor, self-resolving bleeding at the puncture site was the only TBNA-associated complication observed.

Sampling of two lymph node stations versus one was the only variable significantly associated with the likelihood of a positive needle aspirate or biopsy sample (OR $0.15,95 \%$ CI $0.02-0.79, P=.02$ ) at univariate analysis (Figure 1), and it was confirmed as an independent predictor of significance at multivariate analysis (OR $0.12,95 \%$ CI $0.02-0.70$, $P=.01$; Figure 2). There was a trend toward statistical significance when analyzing the impact of the operator performing the TBNA and the likelihood of a positive needle aspirate
TABLE 1. Patient-based analysis of transbronchial needle aspiration results

\begin{tabular}{lcc}
\hline \multicolumn{1}{c}{ Sample type } & Adequate samples & Diagnostic samples \\
\hline $\begin{array}{l}\text { Histologic plus } \\
\text { cytologic }\end{array}$ & $50 / 53(94 \%)$ & $42 / 53(79 \%)$ \\
Cytologic & $50 / 53(94 \%)$ & $42 / 53(79 \%)$ \\
Histologic & $27 / 53(51 \%)$ & $16 / 53(30 \%)$ \\
\hline
\end{tabular}

at both univariate (OR $0.29,95 \% \mathrm{CI} 0.07-1.16, P=.08)$ and multivariate (OR $0.22,95 \%$ CI $0.04-1.04, P=.057$ ) analyses. Because a TBNA cytologic sample representative of the lymph node tissue was obtained in almost all cases (50/53, $94 \%$ ), the adequacy of cytologic samples was not included in the analysis. No correlation was seen between the likelihood of a positive needle aspiration or biopsy sample and patient age and sex, sarcoidosis stage, size and location of sampled nodes, number of needle passes of sampled nodes, or adequacy of histologic TBNA samples.

\section{Discussion}

This study of a large prospective series of patients with sarcoidosis who underwent TBNA suggests that the method has excellent diagnostic yield when hilar or mediastinal lymph node enlargement is present, regardless of stage. This observation, previously described by Wang and colleagues ${ }^{11}$ in a smaller series, is of particular importance because it contradicts earlier studies that reported a far lower yield for TBNA predominantly in stage $\mathrm{II}^{12-15}$ leading some authors to hypothesize that stage II sarcoidosis lymph nodes could have a lower density of granulomas than in stage I. ${ }^{15,26}$ Several limitations of previous studies, such as retrospective design, ${ }^{14,15}$ exclusive acceptance of histologic TBNA samples as diagnostic criteria, ${ }^{12,13}$ and lack of CT guidance to map lymph node areas to be sampled, ${ }^{13}$ may have contributed to the unsatisfactory yield reported with TBNA in stage II sarcoidosis.

In our study, sampling of two lymph node stations versus one was significantly associated with the likelihood of a positive needle aspirate or biopsy sample, and it was shown to be an independent predictor of significance in the multivariate model (Figures 1 and 2). A diagnosis of sarcoidosis was obtained in $92.5 \%$ of patients who had two nodes sampled, as compared with $65 \%$ who had only one, regardless of stage. We therefore recommend sampling more than one nodal station in patients with suspected sarcoidosis whenever possible. It would be important to assess in future studies, however, whether the use of ROSE could help to reduce the number of biopsy sites in patients with a strong clinical likelihood of sarcoidosis ${ }^{10}$ In light of the excellent yield of cytologic material in sarcoidosis. ${ }^{16,22,23,24-26}$ ROSE might in fact obviate the need for further diagnostic sampling by showing nonnecrotizing epithelioid granulomas. ${ }^{27,28}$ 
TABLE 2. Procedure-based analysis of transbronchial needle aspiration results according to American Thoracic Society lymph node station

\begin{tabular}{lccccc}
\hline & All stations & Subcarinal (7) & $\begin{array}{c}\text { Right } \\
\text { paratracheal (4R) }\end{array}$ & $\begin{array}{c}\text { Left } \\
\text { paratracheal (4L) }\end{array}$ & $\begin{array}{c}\text { Right hilar } \\
\text { (10R-11R) }\end{array}$ \\
\hline $\begin{array}{l}\text { Procedures (No.) } \\
\text { Diagnostic samples (No.) }\end{array}$ & 80 & 41 & 30 & 1 & 8 \\
$\quad$ Histologic plus cytologic & $58 / 80(72.5 \%)$ & $30 / 41(73 \%)$ & $23 / 30(77 \%)$ & $0 / 1(0 \%)$ & $5 / 8(62.5 \%)$ \\
$\quad$ Cytologic & $56 / 80(70 \%)$ & $29 / 41(71 \%)$ & $22 / 28(78.5 \%)$ & $0 / 1(0 \%)$ & $5 / 8(62.5 \%)$ \\
$\quad$ Histologic & $18 / 80(22.5 \%)$ & $12 / 41(29 \%)$ & $4 / 28(14 \%)$ & $0 / 1(0 \%)$ & $2 / 6(33 \%)$ \\
Adequate samples & & & & & \\
$\quad$ Cytologic & $70 / 80(87.5 \%)$ & $36 / 41(88 \%)$ & $27 / 30(90 \%)$ & $0 / 1(0 \%)$ & $7 / 8(87.5 \%)$ \\
$\quad$ Histologic & $29 / 80(36 \%)$ & $17 / 41(41 \%)$ & $8 / 30(27 \%)$ & $0 / 1(0 \%)$ & $4 / 8(50 \%)$ \\
\hline
\end{tabular}

We also noted a correlation, although not of statistical significance, between the operator and the TBNA yield at both univariate and multivariate analyses (Figures 1 and 2). Although both of the bronchoscopists involved in the study have long-term experience with TBNA, ${ }^{8,15,16}$ which is the first-step technique for diagnosing hilar and mediastinal lymphadenopathy at our institution, their diagnostic yields were different ( $86 \%$ vs $65 \%$ ). This is not surprising, because TBNA is probably the most operator-dependent bronchoscopic sampling procedure, ${ }^{6}$ and its results have been shown to change in time with experience and practice. ${ }^{29}$

When examining the results of TBNA according to the American Thoracic Society lymph node station, the best success rates were obtained in our series from aspirates or biopsies performed in the right paratracheal $(77 \%)$ and subcarinal $(73 \%)$ areas. Interestingly, enlarged lymph nodes in at least one of these two stations were found by CT scan in the vast majority of cases $(50 / 53,94 \%)$, allowing us to perform most TBNAs in those areas $(71 / 80,89 \%)$. It is likely that this high prevalence of enlarged right paratracheal and subcarinal nodes contributed to the high yield that we achieved, because sampling from those areas has consistently been associated with the best TBNA results in the settings of both neoplastic and nonneoplastic disorders. ${ }^{6,8,15,16,20,21}$ This may also explain in part why we obtained good results even though we used a blind technique. On the other hand, recent studies suggest that guiding TBNA with real-time imaging devices such as ultrasounds might further improve the diagnostic yield of TBNA [24-26].

We obtained rates of successful lymph node sampling of $94 \%$ in the patient-based analysis (Table 1) and $72.5 \%$ in the procedure-based analysis (Table 2), even though we limited the number of needle passes for each sampled node to three at the most. The ability to achieve a diagnosis with a low number of needle passes $(\leq 3)$, which is critical to bronchoscopy time management, has already been demonstrated in studies evaluating TBNA in the mediastinal staging of lung cancer. ${ }^{7}$ Recently, Diacon and associates ${ }^{30}$ showed that three transbronchial needle passes are appropriate when only a tissue diagnosis is sought and when alternative sites or sampling modalities are available. Limiting TBNA performance time yet preserving its yield is of particular

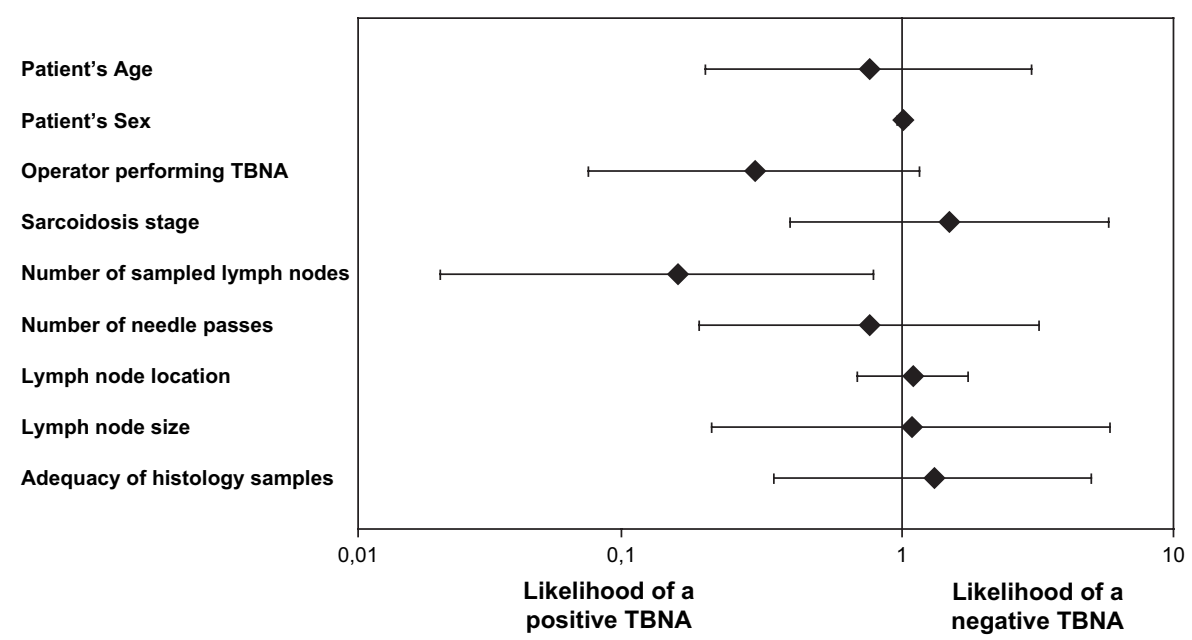

Figure 1. Univariate analysis showing odds ratios of predictor variables of transbronchial needle aspiration (TBNA) yield. 


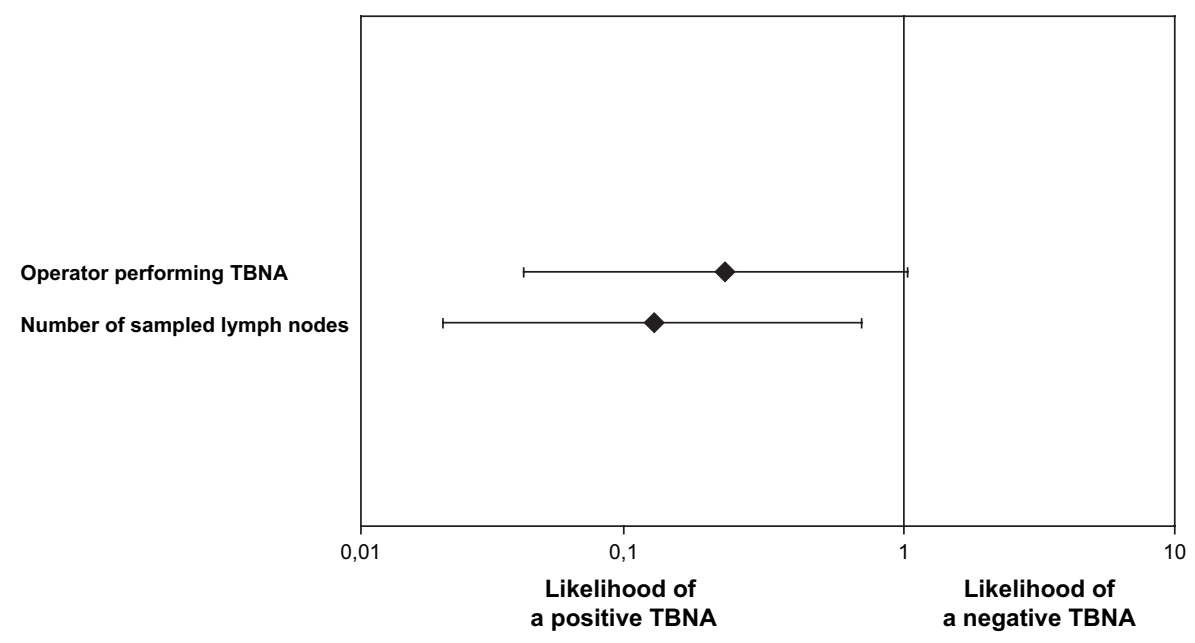

Figure 2. Multivariate analysis showing odds ratios of predictor variables of transbronchial needle aspiration (TBNA) yield.

importance in sarcoidosis, because a combined approach including TBNA and TBLB increases the diagnostic success in this setting, ${ }^{12-16}$ and it is warranted in the absence of ROSE. This observation is confirmed in our study, in which the combination of TBLB and TBNA was associated with a higher diagnostic yield than was TBNA alone (89\% vs $79 \%$ ). Remarkably, however, our study demonstrates that TBNA is superior to TBLB for patients with sarcoidosis seen with hilar or mediastinal lymphadenopathy (yields of $79 \%$ vs $55 \%$, respectively), especially in stage I of the disease (82\% vs $41 \%$, respectively). ${ }^{15,16}$

Unlike repeated observations in the mediastinal staging of lung cancer, we did not find a correlation between the node size in its short-axis diameter and TBNA yield. This observation likely reflects the uniformly large lymph nodes (mean \pm $\mathrm{SD} 1.85 \pm 0.43 \mathrm{~cm}$ ) in our patients, a feature common to sarcoidosis series. $^{24}$

In this study, the diagnostic contribution of cytologic TBNA material was higher than that of histologic samples in both the patient-based (79\% vs $30 \%)$ and the procedurebased analyses ( $70 \%$ vs $22.5 \%$ ), a finding that contrasts with reports from other investigators and our own previous experience. Morales and coworkers ${ }^{12}$ and Wang and colleagues ${ }^{11}$ were able to obtain diagnostic tissue in $51 \%$ and $90 \%$ of patients with sarcoidosis, respectively, with the 19gauge histology needle. In a previous series of 32 patients with stage I sarcoidosis, we obtained adequate and diagnostic histologic TBNA specimens in $64 \%$ and $61 \%$ of procedures, respectively. ${ }^{16}$ With the growing experience of pathologists in the interpretation of TBNA cytologic material in the setting of lung cancer, we have increased the use of 22-gauge cytology needles in the last few years, with satisfactory results. This may have impaired our ability to use large-bore histology needles, leading to inappropriate specimen retrieval or recovery of inadequate samples. On the other hand, a growing body of literature suggests that the demon- stration of nonnecrotizing epithelioid granulomas on cytologic material is feasible and reliable. ${ }^{16,22,23,24-26,28}$ In this particular study, TBNA cytology showed nonnecrotizing granulomas in every single patient who had a positive TBNA histologic study result (16/16 patients). We believe, however, that an attempt to obtain a histologic core tissue with a 19-gauge needle should always be made in patients with suspected sarcoidosis, because histologic evaluation is more reliable in the exclusion of alternative diagnosis, such as lymphoproliferative disorders and tuberculosis. ${ }^{16,22}$

Some limitations to this study need to be assessed to place it into proper context. Although an alternative diagnosis was demonstrated in $13 \%$ of patients $(8 / 61)$, the pretest probability of our study population was high, and TBNA was used in most cases to confirm a strong clinical expectation of sarcoidosis. Our results therefore may not be readily reproducible in an unselected group of patients with hilar or mediastinal lymphadenopathy. We performed most of our procedures in lymph node stations (right paratracheal and subcarinal) that are known to yield the best results with standard TBNA. We are well aware that this choice of biopsy site might create a bias, but we considered the selection of enlarged nodes from areas that are known to be associated with unsatisfactory results with standard TBNA to be ethically unjustifiable. On the other hand, we believe it is important for the community of thoracic surgeons and interventional pulmonologists to appreciate that more than $90 \%$ of patients with suspected sarcoidosis may have lymphadenopathy in areas that are likely to be successfully sampled with standard TBNA.

In conclusion, standard TBNA allows successful lymph node sampling in most patients with sarcoidosis with hilar or mediastinal lymph node enlargement, and it is associated with a high diagnostic yield regardless of the sarcoidosis stage. Sampling of more than one lymph node station, whenever possible, is recommended to increase the likelihood of 
a positive diagnosis. Mediastinoscopy should be reserved for patients with negative TBNA findings.

We thank Dr Adriano Tonelli for his thoughtful review of the manuscript and Drs Valter Merighi, Anna Maspero, and Claudio Nassetti for their contribution of patient data to this series.

\section{References}

1. Venissac N, Alifano M, Mouroux J. Video-assisted mediastinoscopy: experience from 240 consecutive cases. Ann Thorac Surg. 2003;76: 208-12.

2. Reich JM, Brouns MC, O'Connor EA, Edwards MJ. Mediastinoscopy in patients with presumptive stage I sarcoidosis. A risk/benefit, cost/benefit analysis. Chest. 1998;113:147-53.

3. Wang KP, Terry PB. Transbronchial needle aspiration in the diagnosis and staging of bronchogenic carcinoma. Am Rev Respir Dis. 1983; 127:344-7.

4. Toloza EM, Harpole L, Detterbeck F, McCrory DC. Invasive staging of non-small cell lung cancer: a review of the current evidence. Chest. 2003;123(1 Suppl):157S-66.

5. Holty JC, Kushner WG, Gould MK. Accuracy of transbronchial needle aspiration for mediastinal staging of non-small cell lung cancer: a metaanalysis. Thorax. 2005;60:949-55.

6. Harrow EM, Abi-Saleh W, Blum J, Harkin T, Gasparini S, AddrizzoHarris DJ, et al. The utility of transbronchial needle aspiration in the staging of bronchogenic carcinoma. Am J Respir Crit Care Med. 2000;161:601-7.

7. Chin R Jr, McCain TW, Lucia MA, Cappellari JO, Adair NE, Lovato JF, et al. Transbronchial needle aspiration in diagnosing and staging lung cancer: how many aspirates are needed? Am J Respir Crit Care Med. 2002;166:377-81.

8. Patelli M, Agli LL, Poletti V, Trisolini R, Cancellieri A, Lacava N, et al. Role of fiberscopic transbronchial needle aspiration in the staging of N2 disease due to non-small cell lung cancer. Ann Thorac Surg. 2002;73: 407-11.

9. Schenk DA, Chambers SL, Derdak S, Komadina KH, Pickard JS, Strollo PJ, et al. Comparison of the Wang 19-gauge and 22-gauge needles in the mediastinal staging of lung cancer. Am Rev Respir Dis. 1993; 147:1251-8.

10. Diette GB, White P, Terry P, Jenckes M, Rosenthal D, Rubin HR, et al. Utility of rapid on-site cytopathology assessment for bronchoscopic evaluation of lung masses and adenopathy. Chest. 2000;117:1186-90.

11. Wang KP, Johns CJ, Fuenning C, Terry PB. Flexible transbronchial needle aspiration for the diagnosis of sarcoidosis. Ann Otol Rhinol Laryngol. 1989;98:298-300.

12. Morales CF, Patefield AJ, Strollo PJ Jr, Schenk DA. Flexible transbronchial needle aspiration in the diagnosis of sarcoidosis. Chest. 1994;106: 709-11.

13. Leonard C, Tormey VJ, O'Keane CO, Burke CM. Bronchoscopic diagnosis of sarcoidosis. Eur Respir J. 1997;10:2722-4.
14. Bilaçeroğlu S, Perim K, Günel O, Cağirici U, Büyükşsirin M. Combining transbronchial aspiration with endobronchial and transbronchial biopsy in sarcoidosis. Monaldi Arch Chest Dis. 1999;54:217-23.

15. Trisolini R, Lazzari Agli L, Cancellieri A, Poletti V, Candoli P, Paioli D, et al. Transbronchial needle aspiration improves the diagnostic yield of bronchoscopy in sarcoidosis. Sarcoidosis Vasc Diffuse Lung Dis. 2004; 21:147-51.

16. Trisolini R, Lazzari Agli L, Cancellieri A, Poletti V, Tinelli C, Baruzzi $\mathrm{C}$, et al. The value of flexible transbronchial needle aspiration in the diagnosis of stage I sarcoidosis. Chest. 2003;124:2126-30.

17. Statement on sarcoidosis. Joint Statement of the American Thoracic Society (ATS), the European Respiratory Society (ERS) and the World Association of Sarcoidosis and Other Granulomatous Disorders (WASOG) adopted by the ATS Board of Directors and by the ERS Executive Committee, February 1999. Am J Respir Crit Care Med. 1999;160:736-55.

18. Mehta AC, Kavuru MS, Meeker DP, Gephardt GN, Nunez C. Transbronchial needle aspiration for histology specimens. Chest. 1989;96: 1128-32.

19. Mountain CF, Dresler BM. Regional lymph node classification for lung cancer staging. Chest. 1997;111:1718-23.

20. Bilaçeroğlu S, Günel O, Eriş N, Cağirici U, Mehta AC. Transbronchial needle aspiration in diagnosing intrathoracic tuberculous lymphadenitis. Chest. 2004;126:259-67.

21. Sharafkhaneh A, Baaklini W, Gorin AB, Green L. Yield of transbronchial needle aspiration in diagnosis of mediastinal lesions. Chest. 2003; $124: 2131-5$

22. Bilaçeroğlu S, Mehta AC, Light R. Transbronchial needle aspiration for diagnosis of sarcoidosis. J Bronchol. 2004;11:54-61.

23. Fritscher-Ravens A, Sriram PV, Topalidis T, Hauber HP, Meyer A, Soehendra N, et al. Diagnosing sarcoidosis using endosonographyguided fine needle aspiration. Chest. 2000;118:928-35.

24. Annema JT, Vaselic M, Rabe KF. Endoscopic ultrasound-guided fineneedle aspiration for the diagnosis of sarcoidosis. Eur Respir J. 2005; 25:405-9.

25. Wildi SM, Judson MA, Fraig M, Fickling WE, Schmulewitz N, Varadarajulu $\mathrm{S}$, et al. Is endosonography guided fine needle aspiration (EUS-FNA) as good as we think? Thorax. 2004;59:794-9.

26. Wong M, Yasufuku K, Nakajima T, Herth FJ, Sekine J, Shibuya K, et al. Endobronchial ultrasound: new insight for the diagnosis of sarcoidosis Eur Respir J. 2007;29:1182-6.

27. Baram D, Garcia RB, Richman PS. Impact of rapid on-site cytologic evaluation during transbronchial needle aspiration. Chest. 2005;128:869-75.

28. Khoo KL, Ho KY, Nilsson B, Lim KT. EUS-guided FNA immediately after unrevealing transbronchial needle aspiration in the evaluation of mediastinal lymphadenopathy: a prospective study. Gastrointest Endosc. 2006;63:215-20.

29. Haponik EF, Cappellari JO, Chin R, Adair NE, Lykens M, Altford PT, et al. Education and experience improve transbronchial needle aspiration performance. Am J Respir Crit Care Med. 1995;151:1998-2002.

30. Diacon AH, Schuurmans MM, Theron J, Brundyn K, Louw M, Wright CA, et al. Transbronchial needle aspirate: how many passes per target site? Eur Respir J. 2007;29:112-6. 